\title{
Hochschild Cohomology Theories in White Noise Analysis ${ }^{\star}$
}

\author{
Rémi LÉANDRE
}

Institut de Mathématiques de Bourgogne, Université de Bourgogne, 21000, Dijon, France E-mail: Remi.leandre@u-bourgogne.fr

Received June 18, 2008, in final form September 08, 2008; Published online September 27, 2008

Original article is available at http://www.emis.de/journals/SIGMA/2008/066/

\begin{abstract}
We show that the continuous Hochschild cohomology and the differential Hochschild cohomology of the Hida test algebra endowed with the normalized Wick product are the same.
\end{abstract}

Key words: white noise analysis; Hochschild cohomology

2000 Mathematics Subject Classification: 53D55; 60H40

\section{Introduction}

Hochschild cohomology is a basic tool in the deformation theory of algebras. Gerstenhaber has remarked that in his seminal work (we refer to [15] and references therein for that). Deformation quantization $[2,3]$ in quantum field theory leads to some important problems $[10,11,14,40]$. Motivated by that, Dito [12] has defined the Moyal product on a Hilbert space. It is easier to work with models of stochastic analysis although they are similar to models of quantum field. In order to illustrate the difference between these two theories, we refer to:

- The paper on Dirichlet forms in infinite dimensions of Albeverio-Hoegh-Krohn [1] which used measures on the space of distributions, the traditional space of quantum field theory.

- The seminal paper of Malliavin [28] which used the traditional Brownian motion and the space of continuous functions as a topological space. This allows Malliavin to introduce stochastic differential equations in infinite-dimensional analysis, and to interpret some traditional tools of quantum field theory in stochastic analysis.

This remark lead Dito and Léandre [13] to construct of the Moyal product on the Malliavin test algebra on the Wiener space.

It is very classical in theoretical physics [9] that the vacuum expectation of some operator algebras on some Hilbert space is formally represented by formal path integrals on the fields. In the case of infinite-dimensional Gaussian measure, this isomorphism is mathematically well established and is called the Wiener-Itô-Segal isomorphism between the Bosonic Fock space and the $L^{2}$ of a Gaussian measure. The operator algebra is the algebra of annihilation and creation operators with the classical commutation relations. In the case of the classical Brownian motion $B_{t}$ on $\mathbb{R}, B_{t}$ is identified with $A_{t}+A_{t}^{*}$ where $A_{t}$ is the annihilation operator associated to $1_{[0, t]}$ and $A_{t}^{*}$ the associated creation operator.

Let us give some details on this identification [31]. Let $H$ be the Hilbert space of $L^{2}$ maps from $\mathbb{R}^{+}$into $\mathbb{R}$. We consider the symmetric tensor product $\hat{H}^{\otimes n}$ of $H$. It can be realized as the set of maps $h^{n}$ from $\left(\mathbb{R}^{+}\right)^{n}$ into $\mathbb{R}$ such that $\int_{\left(\mathbb{R}^{+}\right)^{n}}\left|h^{n}\left(s_{1}, \ldots, s_{n}\right)\right|^{2} d s_{1} \cdots d s_{n}=\left\|h^{n}\right\|^{2}<\infty$. Moreover these maps $h^{n}\left(s_{1}, \ldots, s_{n}\right)$ are symmetric in $\left(s_{1}, \ldots, s_{n}\right)$. The symmetric Fock space $W_{0}$

\footnotetext{
${ }^{\star}$ This paper is a contribution to the Special Issue on Deformation Quantization. The full collection is available at http://www.emis.de/journals/SIGMA/Deformation_Quantization.html
} 
coincides with the set of formal series $\sum h^{n}$ such that $\sum n !\left\|h^{n}\right\|^{2}<\infty$. The annihilation operators $A_{t}$ and the creation operators are densely defined on $W_{0}$, mutually adjoint and therefore closable. To $h^{n}$ we associate the multiple Wiener chaos $I^{n}\left(h^{n}\right)$

$$
I^{n}\left(h^{n}\right)=\int_{\left(\mathbb{R}^{+}\right)^{n}} h^{n}\left(s_{1}, \ldots, s_{n}\right) \delta B_{s_{1}} \cdots \delta B_{s_{n}}
$$

where $s \rightarrow B_{s}$ is the classical Brownian motion on $\mathbb{R}$. $E_{d P}\left[\left|I^{n}\left(h^{n}\right)^{2}\right|\right]$ for the law of the Brownian motion $d P$ is $n !\left\|h^{n}\right\|^{2}$. $I^{n}\left(h^{n}\right)$ and $I^{m}\left(h^{m}\right)$ are mutually orthogonal on $L^{2}(d P)$. If $F$ belongs to $L^{2}(d P), F$ can be written in a unique way $F=\sum I^{n}\left(h^{n}\right)$ where $\sum h^{n}$ belongs to the symmetric Fock space $W_{0}$. This identification, called chaotic decomposition of Wiener functionals, realizes an isometry between $L^{2}(d P)$ and the symmetric Fock space. $B_{t}$ can be assimilated to the densely defined closable operator on $L^{2}(d P)$

$$
F \rightarrow B_{t} F \text {. }
$$

This operator is nothing else but the operator $A_{t}+A_{t}^{*}$ on the symmetric Fock space.

White noise analysis [18] is concerned with the time derivative of $B_{t}$ (the white noise) as a distribution (an element of $W_{-\infty}$ ) acting on some weighted Fock space $W_{-\infty}$ (we refer to $[4,19,35]$ for textbooks on white noise analysis). Let us recall namely that the Brownian motion is only continuous! The theory of Hida distribution leads to new insight in stochastic analysis.

One of the main points of interest in the white noise analysis is that we can compute the elements of $L\left(W_{\infty_{-}}, W_{-\infty}\right)[20,21,35]$ in terms of kernels. We refer to $[21,27,29]$ for a well established theory of kernels on the Fock space. This theory was motivated by the heuristic constructions of quantum field theory $[5,16,17]$. Elements of $L\left(W_{\infty_{-}}, W_{-\infty}\right)$ can be computed in a sum of multiple integrals of the elementary creation and annihilation operators.

This theorem plays the same role as the theorem of Pinczon [37, 38]: the operators acting on $\mathbb{C}\left(x_{1}, \ldots, x_{d}\right)$, the complex polynomial algebra on $\mathbb{R}^{d}$, are series of differential operators with polynomial components. This theorem of Pinczon allowed Nadaud [32, 33] to show that the continuous Hochschild cohomology on $C^{\infty}\left(\mathbb{R}^{d}\right)$ is equal to the differential Hochschild cohomology of the same algebra (we refer to papers of Connes [8] and Pflaum [36] for other proofs).

In the framework of white noise analysis we have an analogous theorem to the theorem of Pinczon [37]. Therefore we can repeat in this framework the proof of Nadaud. We show that the continuous Hochschild cohomology [22] of the Hida Fock space (we consider series of kernels) is equal to the differential Hochschild cohomology (we consider finite sums of kernels).

In the first part of this work, we recall the theorem of Obata which computes the operators on the Hida Fock space: Obata considers standard creation operators and standard annihilation operators. We extend this theorem in the second part to continuous multilinear operators on the Hida algebra, endowed with the normalized Wick product. This Hida test algebra was used by Léandre $[23,24]$ in order to define some star products in white noise analysis.

We refer to the review paper of Léandre for deformation quantization in infinite-dimensional analysis [25].

\section{A brief review on Obata's theorem}

We consider the Hilbert space $H=L^{2}(\mathbb{R}, d t)$. We consider the operator $\Delta=1+t^{2}-d^{2} / d t^{2}$. It has eigenvalues $\mu_{j}=(2 j+2)$ associated to the normalized eigenvectors $e_{j}, j \geq 0$. We consider the Hilbert space $H_{k}$ of series $f=\sum \lambda_{j} e_{j}$ such that

$$
\|f\|_{k}^{2}=\sum\left|\lambda_{j}\right|^{2} \mu_{j}^{2 k}<\infty
$$


It is the Sobolev space associated to $\Delta^{k}$. Since $\mu_{j}>1, H_{k} \subseteq H_{k^{\prime}}$ if $k>k^{\prime}$, and the system of Sobolev norms $\|\cdot\|_{k}$ increases when $k$ increases. Therefore we can define the test functional space $H_{\infty-}$ of functions $f$ such that all norms $\|f\|_{k}<\infty$. A functional $F$ with values in this space is continuous if and only if it is continuous for all Sobolev norms $\|\cdot\|_{k}, k>0$,

$$
H_{\infty-}=\cap_{k \geq 0} H_{k}
$$

The topological dual of $H_{\infty-}$ is the space of Schwartz distributions:

$$
H_{-\infty}=\cup_{k<0} H_{k} .
$$

$\sigma$ is called a distribution if the following condition holds: let $f$ be in $H_{\infty}$. For some $k>0$, there exists $C_{k}$ such that for all $f \in H_{\infty_{-}},|\langle\sigma, f\rangle| \leq C_{k}\|f\|_{k}$ Therefore we get a Gel'fand triple

$$
H_{\infty-} \subseteq H \subseteq H_{-\infty}
$$

We complexify all these spaces (We take the same notation). It is important to complexify these spaces to apply Potthoff-Streit theorem [39].

Let $A=\left(\left(i_{1}, r_{1}\right), \ldots,\left(i_{n}, r_{n}\right)\right)$ where $i_{1}<i_{2}<\cdots<i_{n}$ and $r_{i}>0$. We put

$$
|A|=\sum r_{i}
$$

and

$$
e_{A}=\hat{\otimes} e_{i_{j}}^{r_{j}}
$$

where we consider a normalized symmetric tensor product. We introduce the Hida weight

$$
\|A\|=\prod_{\left(i_{j}, r_{j}\right) \in A}\left(2 i_{j}+2\right)^{r_{j}}
$$

We consider the weighted Fock space $W_{k}$ of series

$$
\phi=\sum_{A} \lambda_{A} e_{A}
$$

such that

$$
\|\phi\|_{k}=\sum_{A}\left|\lambda_{A}\right|^{2}\|A\|^{2 k}|A| !<\infty
$$

( $\lambda_{A}$ is complex). These systems of norms increase when $k$ increases.

We consider

$$
W_{\infty-}=\cap_{k>0} W_{k}
$$

endowed with the projective topology and its topological dual (called the space of Hida distributions)

$$
W_{-\infty}=\cup_{k<0} W_{k}
$$

endowed with the inductive topology. We get a Gel'fand triple

$$
W_{\infty-} \subseteq W_{0} \subseteq W_{-\infty},
$$

$W_{0}$ is the classical Fock space of quantum physics.

We consider $\xi \in H_{\infty-}$ and the classical coherent vector

$$
\phi_{\xi}=\sum_{n \in \mathbb{N}} \frac{\xi^{\otimes n}}{n !},
$$

$\phi_{\xi}$ belongs to the Hida test functional space $W_{\infty_{-}}$. 
Definition 1. Let $\Xi$ belong to $L\left(W_{\infty_{-}}, W_{-\infty}\right)$. Its symbol is the function $\hat{\Xi}$ from $H_{\infty-} \times H_{\infty_{-}}$ into $\mathbb{C}$ defined by

$$
\hat{\Xi}(\xi, \eta)=\left\langle\Xi \phi_{\xi}, \phi_{\eta}\right\rangle_{0}
$$

If $\Xi$ belongs to $L\left(W_{\infty_{-}}, W_{-\infty}\right)$, its symbol satisfies clearly the following properties:

$\left(\mathrm{P}_{1}\right)$ For any $\xi_{1}, \xi_{2}, \eta_{1}, \eta_{2} \in H_{\infty-}$, the function

$$
(z, w) \rightarrow \hat{\Xi}\left(z \xi_{1}+\xi_{2}, w \eta_{1}+\eta_{2}\right)
$$

is an entire holomorphic function on $\mathbb{C} \times \mathbb{C}$.

$\left(\mathrm{P}_{2}\right)$ There exists a constant $K$ and a constant $k>0$ such that

$$
|\hat{\Xi}(\xi, \eta)|^{2} \leq K \exp \left[\|\xi\|_{k}^{2}\right] \exp \left[\|\eta\|_{k}^{2}\right]
$$

The converse of this theorem also holds. It is a result of Obata [34] which generalizes the theorem of Potthoff-Streit characterizing distribution in white noise analysis [39]. If a function $\hat{\Xi}$ from $H_{\infty_{-}} \times H_{\infty_{-}}$into $\mathbb{C}$ satisfies $\left(\mathrm{P}_{1}\right)$ and $\left(\mathrm{P}_{2}\right)$, it is the symbol of an element of $L\left(W_{\infty_{-}}, W_{-\infty}\right)$. Its continuity norms can be estimated in a universal way linearly in the data of $\left(\mathrm{P}_{2}\right)$.

This characterization theorem allows Obata to show that an element $\Xi$ of $L\left(W_{\infty_{-}}, W_{-\infty}\right)$ can be decomposed into a sum

$$
\Xi=\sum_{l, m} \Xi_{l, m}\left(k_{l, m}\right)
$$

where $\Xi_{l, m}\left(k_{l, m}\right)$ is defined by the following considerations.

Let $A$ be as given above (1). Let $a_{i}^{*}$ be the standard creation operator

$$
a_{i}^{*} e_{A}=c\left(r_{i}\right) e_{A^{i}}
$$

where

$$
A^{i}=\left(\left(i_{1}, r_{1}\right), \ldots,\left(i_{l}, r_{l}\right),\left(i, r_{i}+1\right),\left(i_{l+1}, r_{l+1}\right), \ldots,\left(i_{n}, r_{n}\right)\right)
$$

if $i_{l}<i<i_{l+1}$. If $i$ does not appear in $A$, we put $r_{i}=1$. We consider the standard annihilation operator $e_{i}$ defined

$$
a_{i} e_{A}=0
$$

if $i$ does not appear in $A$ and equals to $c^{\prime}\left(r_{i}\right) e_{A_{i}}$ where

$$
A_{i}=\left(\left(i_{1}, r_{i}\right), \ldots,\left(i_{l}, r_{l}\right),\left(i, r_{i}-1\right), \ldots,\left(i_{n}, r_{n}\right)\right) .
$$

The constants $c\left(r_{i}\right)$ and $c^{\prime}\left(r_{i}\right)$ are computed in [31]. Their choice is motivated by the use of Hermite polynomial on the associated Gaussian space by the Wiener-Itô-Segal isomorphism: the role of Hermite polynomial in infinite dimensions is played by the theory of chaos decomposition through the theory of multiple Wiener integrals. The annihilation operator $a_{i}$ corresponds to the stochastic derivative in the direction of $e_{i}$ on the corresponding Wiener space. $a_{i}^{*}$ is its adjoint obtained by integrating by parts on the Wiener space. We consider the operator $\Xi_{I, J}$

$$
\phi \rightarrow a_{i_{1}}^{*} \cdots a_{i_{l}}^{*} a_{j_{1}} \cdots a_{j_{m}} \phi=a_{I}^{*} a_{J} \phi
$$


It belongs to $L\left(W_{\infty-}, W_{-\infty}\right)$ and its symbol is [34]

$$
\exp \left[\langle\xi, \eta\rangle_{0}\right] \prod_{j_{k} \in J}\left\langle e_{j_{k}}, \xi\right\rangle_{0} \prod_{i_{k} \in I}\left\langle e_{i_{k}}, \eta\right\rangle_{0} .
$$

Therefore we can consider

$$
\Xi_{l, m}\left(k_{l, m}\right)=\sum_{|I|=l,|J|=m} \lambda_{I, J} a_{I}^{*} a_{J}
$$

where

$$
\sum\left|\lambda_{I, J}\right|^{2}\|I\|^{-k}\|J\|^{-k}<\infty
$$

for some $k>0 . \Xi_{l, m}=\sum \lambda_{I, J} e_{I} \otimes e_{J}$ defines an element of $H_{-\infty}^{\otimes(l+m)} . \Xi_{I, J}$ can be extended by linearty to

$$
\sum \lambda_{I, J} \Xi_{I, J}=\Xi_{l, m}\left(k_{l, m}\right)
$$

$\Xi_{l, m}\left(k_{l, m}\right)$ belongs to $L\left(W_{\infty-}, W_{-\infty}\right)$ if $k_{l, m}$ belongs to $H_{-\infty}^{\otimes(l+m)}$. This last space is $\cup_{k>0} H_{-k}^{\otimes(l+m)}$ endowed with the inductive topology. $\Xi_{l, m}\left(k_{l, m}\right)$ belongs to $L\left(W_{\infty_{-}}, W_{\infty_{-}}\right)$if $k_{l, m}$ belongs to $H_{\infty-}^{\otimes l} \otimes H_{-\infty}^{\otimes m}$. This means that there exists $k$ such that for all $k^{\prime}$

$$
\sum\left|\lambda_{I, J}\right|^{2}\|I\|^{k^{\prime}}\|J\|^{-k}<\infty
$$

for some $k>0$. The symbol of $\Xi_{l, m}\left(k_{l, m}\right)$ satisfies

$$
\hat{\Xi}_{l, m}\left(k_{l, m}\right)(\eta, \xi)=\left\langle k_{l, m}, \eta^{\otimes l} \otimes \xi^{\otimes m}\right\rangle \exp \left[\langle\xi, \eta\rangle_{0}\right] .
$$

Following the heuristic notation of quantum field theory $[5,6,16,17,30]$, the operator $\Xi_{l, m}\left(k_{l, m}\right)$ can be written as

$$
\Xi_{l, m}\left(k_{l, m}\right)=\int_{\mathbb{R}^{l+m}} k\left(s_{1}, \ldots, s_{l}, t_{1}, \ldots, t_{m}\right) a_{s_{1}}^{*} \cdots a_{s_{l}}^{*} a_{t_{1}} \cdots a_{t_{m}} d s_{1} \cdots d s_{l} d t_{1} \cdots d t_{m} .
$$

The "elementary" creation operators $a_{s}^{*}$ and the "elementary" annihilation operators $a_{t}$ satisfy the canonical commutation relations

$$
\left[a_{s}^{*}, a_{t}^{*}\right]=\left[a_{s}, a_{t}\right]=0, \quad\left[a_{s}^{*}, a_{t}\right]=\delta(s-t),
$$

where $\delta(\cdot)$ is the Dirac function in 0 .

\section{Fock expansion of continuous multilinear operators}

We are motivated in this work by the Hochschild cohomology in white noise analysis. For that, we require that $W_{\infty-}$ is an algebra. In order to be self-consistent we will take the model of [24] or $[26]$.

We will take the normalized Wick product

$$
: e_{A} \cdot e_{B}:=e_{A \cup B}
$$

where $A \cup B$ is obtained by concatenating the indices and adding the length of these when the same appears twice. 
$W_{\infty-}$ is not the same space as before. We consider another Hida Fock space. $W_{k, C}$ is the space of $\phi=\sum_{A} \lambda_{A} e_{A}$ such that

$$
\|\phi\|_{k, C}^{2}=\sum_{A}\left|\lambda_{A}\right|^{2} C^{2|A|}\|A\|^{2 k}|A| !<\infty .
$$

$W_{k, C}$ can be identified with the Bosonic Fock space associated to the Hilbert Sobolev space associated to the operator $C \Delta^{k}$. $W_{\infty-}$ is the intersection of $W_{k, C}, k>0, C>0$. This space is endowed with the projective topology.

By a small improvement of [24] and [26], we get:

Theorem 1. $W_{\infty-}$ is a topological algebra for the normalized Wick product.

Proof. The only new ingredient in the proof of $[24,26]$ is that

$$
|A \cup B| ! \leq 2^{|A|+|B|}|A| !|B| !
$$

Let us give some details. Let us consider

$$
\phi^{1}=\sum_{A} \lambda_{A}^{1} e_{A}, \quad \phi^{2}=\sum_{A} \lambda_{A}^{2} e_{A} .
$$

We have

$$
: \phi^{1} \cdot \phi^{2}:=\sum_{A} \mu_{A} e_{A}, \quad \text { where } \quad \mu_{A}=\sum_{B \cup D=A} \lambda_{B}^{1} \lambda_{D}^{2}
$$

There are at most $2^{|A|}$ terms in the previous sum. By Jensen inequality

$$
\left|\mu_{A}\right|^{2} \leq C_{1}^{|A|} \sum_{B \cup D=A}\left|\lambda_{B}^{1}\right|^{2}\left|\lambda_{D}^{2}\right|^{2} .
$$

Therefore

$$
\left\|: \phi^{1} \cdot \phi^{2}:\right\|_{k, C}^{2} \leq \sum_{A}\left(C_{1} C\right)^{2|A|} C^{2|A|}\|A\|^{2 k}|A| ! \sum_{B \cup D=A}\left|\lambda_{B}^{1}\right|^{2}\left|\lambda_{D}^{2}\right|^{2} .
$$

But

$$
\|A\|^{2 k} \leq\|B\|^{2 k}\|D\|^{2 k} \quad \text { and } \quad|A| ! \leq 2^{|B|+|D|}|B| !|D| !
$$

if the concatenation of $B$ and $D$ equals $A$. Therefore, for some $C_{1}$

$$
\left\|: \phi^{1} \cdot \phi^{2}:\right\|_{k, C}^{2} \leq \sum_{A} \sum_{B \cup D=A}\left(C_{1} C\right)^{|B|+|D|}\|B\|^{2 k}\|D\|^{2 k}|B| !|D| ! \leq\left\|\phi^{1}\right\|_{k, C_{1} C}^{2}\left\|\phi^{2}\right\|_{k, C_{1} C}^{2} .
$$

This shows the result.

Let $L\left(W_{\infty_{-}}^{n}, W_{\infty_{-}}\right)$be the space of $n$-multilinear continuous applications from $W_{\infty-}$ into $W_{\infty-}$.

Definition 2. The symbol $\hat{\Xi}$ of an element $\Xi$ of $L\left(W_{\infty_{-}}^{n}, W_{\infty_{-}}\right)$is the map from $H_{\infty_{-}}^{n} \times H_{\infty_{-}}$ into $\mathbb{C}$ defined by

$$
\xi^{1} \times \xi^{2} \times \cdots \times \xi^{n} \times \eta \rightarrow\left\langle\Xi\left(\phi_{\xi^{1}}, \ldots, \phi_{\xi^{n}}\right), \phi_{\eta}\right\rangle_{0}=\hat{\Xi}\left(\xi^{1}, \ldots, \xi^{n}, \eta\right) .
$$


$\Xi$ belongs to $L\left(W_{\infty-}^{n}, W_{\infty-}\right)$ if for any $(k, C)$, there exists $\left(k_{1}, C_{1}, K_{1}\right)$ such that

$$
\left\|\Xi\left(\phi^{1}, \ldots, \phi^{n}\right)\right\|_{k, C} \leq K_{2} \prod\left\|\phi^{i}\right\|_{k_{2}, C_{2}} .
$$

If $\Xi$ belongs to $L\left(W_{\infty_{-}}^{n}, W_{\infty_{-}}\right)$, its symbol satisfies clearly the following properties:

$\left(\mathrm{O}_{1}\right)$ For any elements $\xi_{1}^{1}, \ldots, \xi_{1}^{n}, \xi_{2}^{1}, \ldots, \xi_{2}^{n}, \eta_{1}, \eta_{2}$ of $H_{\infty-}$, the map

$$
\left(z_{1}, \ldots, z_{n}, w\right) \rightarrow \hat{\Xi}\left(z_{1} \xi_{1}^{1}+\xi_{2}^{1}, \ldots, z_{n} \xi_{1}^{n}+\xi_{2}^{n}, w \eta_{1}+\eta_{2}\right)
$$

is an entire holomorphic map from $\mathbb{C}^{n} \times \mathbb{C}$ into $\mathbb{C}$.

$\left(\mathrm{O}_{2}\right)$ For all $k>0, K>0$, there exists numbers $C, k_{1}>k, K_{1}>0$ such that

$$
\left|\hat{\Xi}\left(\xi^{1}, \ldots, \xi^{n}, \eta\right)\right|^{2} \leq C \exp \left[K_{1} \sum_{i=1}^{n}\left\|\xi^{i}\right\|_{k_{1}}^{2}+K\|\eta\|_{-k}^{2}\right] .
$$

We prove the converse of this result. It is a small improvement of the theorem of Ji and Obata [20].

Theorem 2. If a function $\hat{\Xi}$ from $\mathbb{C}^{n} \times \mathbb{C}$ into $\mathbb{C}$ satisfies to $\left(\mathrm{O}_{1}\right)$ and $\left(\mathrm{O}_{2}\right)$, it is the symbol of an element $\Xi$ of $L\left(W_{\infty_{-}}^{n}, W_{\infty_{-}}\right)$. The different modulus of continuity can be estimated in terms of the data in $\left(\mathrm{O}_{2}\right)$.

Proof. It is an adaptation of the proof of a result of Obata [34], the result which was generalizing Potthoff-Streit theorem. We omit all the details. This classical Potthoff-Streit theorem is the following. Let $\Phi$ in $W_{-\infty}$ be the topological dual of $W_{\infty_{-}-}$. We define its $S$-transform as follows

$$
S(\xi)=\left\langle\Phi, \phi_{\xi}\right\rangle_{0}
$$

for $\xi \in H_{-\infty}$. The $S$-transform of $\Phi$ satisfies the following properties:

i) the function $z \rightarrow S\left(z \xi_{1}+\xi_{2}\right)$ is entire holomorphic;

ii) for some $K_{1}>0, K_{2}>0$ and some $k \in \mathbb{R}$

$$
|S(\xi)|^{2} \leq K_{1} \exp \left[K_{2}\|\xi\|_{k}^{2}\right]
$$

Potthoff-Streit theorem states the opposite [20, Lemma 3.2]: if a function $S$ from $H_{\infty-}$ into $\mathbb{C}$ satisfies i) and ii), it is the $S$-transform of a distribution $\Phi$. Moreover, there exists $C$ depending only of $K_{2}$ such that

$$
\|\Phi\|_{-k-r, C}^{2} \leq K_{1}
$$

for all $r>0$.

From this theorem, we deduce that there exists a distribution $\Phi_{\xi^{1}, \ldots, \xi^{n-1}, \eta}$ such that

$$
\hat{\Xi}\left(\xi^{1}, \ldots, \xi^{n}, \eta\right)=\left\langle\Phi_{\xi^{1}, \ldots, \xi^{n-1}, \eta}, \phi_{\xi}\right\rangle_{0} .
$$

Moreover there exists $C$ independent of $\eta, \xi^{1}, \ldots, \xi^{n-1}$ such that

$$
\left\|\Phi_{\xi^{1}, \ldots, \xi^{n-1}, \eta}\right\|_{-k_{1}-r, C}^{2} \leq K_{2} \exp \left[K_{1} \sum_{i=1}^{n-1}\left\|\xi^{i}\right\|_{k_{1}}^{2}+K\|\eta\|_{-k}^{2}\right] \text {. }
$$


If $\phi$ belongs to $W_{\infty-}$ we put

$$
G_{\phi}\left(\xi^{1}, \ldots, \xi^{n-1}, \eta\right)=\left\langle\Phi_{\xi^{1}, \ldots, \xi^{n-1}, \eta}, \phi\right\rangle_{0} .
$$

We have for some $K_{2}, C_{2}, K_{1}, k_{1}, k_{2}$ depending only on the previous datas that

$$
\left\|G_{\phi}\left(\xi^{1}, \ldots, \xi^{n-1}, \eta\right)\right\|^{2} \leq K_{2}\|\phi\|_{k_{2}, C_{2}}^{2} \exp \left[K_{1} \sum_{i=1}^{n-1}\left\|\xi^{i}\right\|_{k_{1}}^{2}+K\|\eta\|_{-k}^{2}\right] .
$$

The two properties $\left(\mathrm{O}_{1}\right)$ and $\left(\mathrm{O}_{2}\right)$ are satisfied at the step $n-1$. By induction, we deduce that

$$
G_{\phi}\left(\xi^{1}, \ldots, \xi^{n-1}, \eta\right)=\hat{\Xi}_{\phi}\left(\xi^{1}, \ldots, \xi^{n-1}, \eta\right) .
$$

Moreover, we get that

$$
G_{\phi}\left(\xi^{1}, \ldots, \xi^{n-1}, \eta\right)=\left\langle\Xi_{\phi}\left(\phi_{\xi^{1}}, \ldots, \phi_{\xi^{n-1}}\right), \phi_{\eta}\right\rangle_{0},
$$

where $\Xi_{\phi}$ is an element of $L\left(W_{\infty_{-}}^{n-1}, W_{\infty_{-}}\right)$depending linearly and continuously from $\phi \in W_{\infty_{-}}$. We put

$$
\Xi\left(\phi^{1}, \ldots, \phi^{n-1}, \phi\right)=\Xi_{\phi}\left(\phi^{1}, \ldots, \phi^{n-1}\right) .
$$

It remains to prove the result for $n=1$. It is a small improvement of the proof of the result of $\mathrm{Ji}$ and Obata [20]. Let us give some details.

By using Potthoff-Streit theorem, we deduce that there is a distribution $\Phi_{\eta}$ such that

$$
\hat{\Xi}(\xi, \eta)=\left\langle\Phi_{\eta}, \phi_{\xi}\right\rangle_{0} .
$$

Moreover there exists $C$ independent of $\eta$ such that

$$
\left\|\Phi_{\eta}\right\|_{-k_{1}-r, C}^{2} \leq K_{2} \exp \left[K\|\eta\|_{-k}^{2}\right]
$$

If $\phi$ belongs to $W_{\infty-}$, we set

$$
G_{\phi}(\eta)=\left\langle\Phi_{\eta}, \phi\right\rangle_{0}
$$

We have

$$
\left|G_{\phi}(\eta)\right|^{2} \leq K_{3} \|\left.\phi\right|_{k_{2}, C_{2}} ^{2} \exp \left[K\|\eta\|_{-k}^{2}\right]
$$

for some $k_{2}>0$. We apply Potthoff-Streit theorem (see [20, Lemma 3.3]). There exists an element $\Xi(\phi)$ of $W_{k-r, C}$ where $k>0$ depending continuously of $\phi$ such that

$$
G_{\phi}(\eta)=\left\langle\Xi(\phi), \phi_{\eta}\right\rangle \text {. }
$$

We have clearly

$$
\left\langle\Xi\left(\phi_{\xi}\right), \phi_{\eta}\right\rangle=\hat{\Xi}(\xi, \eta) \text {. }
$$

This shows the result.

The following statements follow closely [7, Appendix].

Let $\Xi$ be an element of $L\left(W_{\infty-}^{n}, W_{\infty_{-}}\right)$. Let $\hat{\Xi}$ be its symbol. We put:

$$
\Psi\left(\xi^{1}, \ldots, \xi^{n}, \eta\right)=\exp \left[-\sum_{i=1}^{n}\left\langle\xi_{i}, \eta\right\rangle_{0}\right] \hat{\Xi}\left(\xi^{1}, \ldots, \xi^{n}, \eta\right) .
$$


Clearly $\Psi$ satisfies to $\left(\mathrm{O}_{1}\right)$ and $\left(\mathrm{O}_{2}\right)$. We put

$$
\begin{aligned}
& \psi\left(z_{1}^{1}, \ldots, z_{m_{1}}^{1}, z_{1}^{2}, \ldots, z_{m_{2}}^{2}, \ldots, z_{1}^{n}, \ldots, z_{m_{n}}^{n}, w_{1}, \ldots, w_{l}\right) \\
& \quad=\Psi\left(z_{1}^{1} \xi_{1}^{1}+\cdots+z_{m_{1}}^{1} \xi_{m_{1}}^{1}, \ldots, z_{1}^{n} \xi_{1}^{n}+\cdots+z_{m_{n}}^{n} \xi_{m_{n}}^{n}, w_{1} \eta_{1}+\cdots+w_{l} \eta_{l}\right) .
\end{aligned}
$$

We put $M=\left(m_{1}, \ldots, m_{n}\right)$ and

$$
\begin{aligned}
K_{l, M} & \left(\xi_{1}^{1}, \ldots, \xi_{m_{1}}^{1}, \ldots, \xi_{1}^{n}, \ldots, \xi_{m_{n}}^{n}, \eta_{1}, \ldots, \eta_{l}\right) \\
& =\frac{1}{l ! m_{1} ! \cdots m_{n} !} \frac{\partial^{l+\sum m_{i}}}{\partial z_{1}^{1} \cdots \partial z_{m_{1}}^{1} \cdots \partial z_{1}^{n} \cdots \partial z_{m_{n}}^{n} \partial w_{1} \cdots \partial w_{l}} \psi(0,0, \ldots, 0) .
\end{aligned}
$$

$K_{L, M}$ is an $l+\sum m_{i}$ multilinear map.

Since $\psi$ is holomorphic, we have a Cauchy type representation of the considered expression $K_{l, M}\left(\xi_{1}^{1}, \ldots, \xi_{m_{1}}^{1}, \ldots, \xi_{1}^{n}, \ldots, \xi_{m_{n}}^{n}, \eta_{1}, \ldots, \eta_{l}\right)$

$$
\begin{aligned}
K_{l, M} & \left(\xi_{1}^{1}, \ldots, \xi_{m_{1}}^{1}, \ldots, \xi_{1}^{n}, \ldots, \xi_{m_{n}}^{n}, \eta_{1}, \ldots, \eta_{l}\right)=\frac{1}{l ! m_{1} ! \cdots m_{n} !} \prod_{j=1}^{n} \prod_{i=1}^{m_{j}} \frac{1}{2 \pi} \int_{\left|z_{i}^{j}\right|=r_{i}^{j}} \frac{\left|d z_{i}^{j}\right|}{\left(z_{i}^{j}\right)^{2}} \\
& \times \prod_{k=1}^{l} \int_{\left|w_{k}\right|=s_{k}} \frac{\left|d w_{k}\right|}{w_{k}^{2}} \psi\left(z_{1}^{1}, \ldots, z_{m_{1}}^{1}, \ldots, z_{1}^{n}, \ldots, z_{m_{n}}^{n}, w_{1}, \ldots, w_{l}\right) .
\end{aligned}
$$

We deduce from $(\mathrm{O})_{2}$ a bound of $K_{l, M}$ of the type (D.5) in [7]

$$
\begin{aligned}
& \left|K_{l, M}\left(\xi_{1}^{1}, \ldots, \xi_{m_{1}}^{1}, \ldots, \xi_{1}^{n}, \ldots, \xi_{m_{n}}^{n}, \eta_{1}, \ldots, \eta_{l}\right)\right| \leq \frac{C}{l ! \prod m_{i} !} \frac{1}{r_{1}^{1} \cdots r_{m_{1}}^{1} \cdots r_{1}^{n} \cdots r_{m_{n}}^{n} s_{1} \cdots s_{l}} \\
& \quad \times \exp \left[K_{1}\left\{\sum_{i, j} r_{i}^{j}\left\|\xi_{i}^{j}\right\|_{k_{1}}\right\}^{2}\right] \exp \left[K\left\{\sum_{j} s_{j}\left\|\eta_{j}\right\|_{-k}\right\}^{2}\right]
\end{aligned}
$$

for some $k_{1}>k$ and $K, k>0$ and some $K_{1}>0$.

According to [7, (D.6)], we choose

$$
r_{i}^{j}=\frac{R}{C m_{j}\left\|\xi_{i}^{j}\right\|_{k_{1}}}, \quad s_{j}=\frac{S}{C l\left\|\eta_{j}\right\|_{-k}}
$$

and we deduce a bound of $K_{l, M}$ in

$$
\frac{C}{l ! \prod m_{i} !} \prod\left(\frac{C m_{i}}{R}\right)^{m_{i}}\left(\frac{C l}{S}\right)^{l} \prod\left\|\xi_{i}^{j}\right\|_{k_{1}} \prod\left\|\eta_{i}\right\|_{-k} \exp \left[K R^{2}\right] \exp \left[K S^{2}\right]
$$

Clearly, $K_{l, M}$ is a multilinear application in $\xi_{i}^{j}, w_{k}$. By (2), $K_{l, M}$ is continuous. Therefore $K_{l, M}$ can be identified with an element of $H_{\infty-}^{\otimes l} \otimes H_{-\infty}^{\otimes \sum_{i}}$. We consider

$$
\hat{\Xi}_{l, M}\left(\xi^{1}, \ldots, \xi^{n}, \eta\right)=K_{l, M}\left(\xi^{1}, \ldots, \xi^{1}, \xi^{2}, \ldots, \xi^{2}, \ldots, \xi^{n}, \ldots, \xi^{n}, \eta, \ldots, \eta\right),
$$

where $\xi_{i}$ is taken $m_{i}$ times and $\eta l$ times.

By holomorphy,

$$
\hat{\Xi}=\sum \hat{\Xi}_{l, M} \exp \left[\sum_{i=1}^{n}\left\langle\xi^{i}, \eta\right\rangle_{0}\right] .
$$


and the series converges in the sense of $\left(\mathrm{O}_{1}\right)$ and $\left(\mathrm{O}_{2}\right)$. Only the second statement presents some difficulties. We remark for that by [7, page 557] $\left(\alpha(n)=1, G_{\alpha}(s)=\exp [s]\right)$

$$
\inf _{s>0} \exp [s] s^{-n} \leq C n ! n^{-2 n}
$$

We deduce a bound analog to the bound (D.7) in [7]

$$
\left|\hat{\Xi}_{l, M}\right|^{2} \leq \frac{1}{\left(l^{l} \prod m_{i}^{m_{i}}\right)} C^{l} \prod C^{m_{i}} \exp \left[D \sum\left\|\xi^{i}\right\|_{k_{1}}^{2}+D_{1}\|\eta\|_{-k}^{2}\right] .
$$

$x^{n} \exp \left[-D_{1} x^{2}\right]$ has a bound in $\exp \left[-C_{1} n\right] C^{n} n^{n / 2}$. If $D_{1}$ is large, $C$ can be chosen very small and $C_{1}$ very large. We deduce the following bound

$$
\left|\hat{\Xi}_{l, M}\right|^{2} \leq C^{l} C^{\sum m_{i}} \exp \left[-C_{1} l-C_{1} \sum m_{i}\right] \exp \left[D_{2} \sum\left\|\xi^{i}\right\|_{k_{1}}^{2}+D_{2}\|\eta\|_{-k}^{2}\right] .
$$

We remark if $D_{2}$ is very large that $C$ can be chosen very small and $C_{1}$ can be chosen very large. We remark if $C_{1}$ is large

$$
\sum_{l, M} \exp \left[-C_{1} l-C_{1} \sum m_{i}\right]<\infty
$$

in order to see that the series $\Xi_{l, M}$ converges in $L\left(W_{\infty_{-}}^{n}, W_{\infty_{-}}\right)$.

Definition 3. The series $\sum_{l, M} \Xi_{l, M}=\Xi$ is called the Fock expansion of the element $\Xi$ belonging to $L\left(W_{\infty-}^{n}, W_{\infty-}\right)$.

\section{Isomorphism of Hochschild cohomology theories}

In this part, we prove the main theorem of this work.

Lemma 1. If $\xi$ belongs to $H_{\infty-}$,

$$
\xi^{\otimes n}=: \xi:^{n} .
$$

Proof. We put $\xi=\sum \lambda_{i} e_{i}$ such that

$$
\xi^{\otimes n}=\sum_{i_{1}, \ldots, i_{n}} \lambda_{i_{1}} \cdots \lambda_{i_{n}} e_{i_{1}} \otimes_{0} \cdots \otimes_{0} e_{i_{n}}
$$

where $\otimes_{0}$ denotes the traditional tensor product. By regrouping various element, we deduce that

$$
\begin{aligned}
\xi^{\otimes n} & =\sum_{\substack{i<1<\cdots<i_{r} ; n_{1} \neq 0, \ldots, n_{r} \neq 0 ; \\
n_{1}+\cdots+n_{r}=n}} \lambda_{i_{1}}^{n_{1}} \cdots \lambda_{i_{r}}^{n_{r}} \frac{n !}{n_{1} ! \cdots n_{r} !} e_{i_{1}}^{\otimes n_{1}} \hat{\otimes} \cdots \hat{\otimes} e_{i_{r}}^{n_{r}} \\
& =\sum_{\substack{i<1<\cdots<i_{r} ; n_{1} \neq 0, \ldots, n_{r} \neq 0 ; \\
n_{1}+\cdots+n_{r}=n}} \lambda_{i_{1}}^{n_{1}} \cdots \lambda_{i_{r}}^{n_{r}} \frac{n !}{n_{1} ! \cdots n_{r} !}: e_{i_{1}}:{ }^{\otimes n_{1}} \cdots: e_{i_{r}}:{ }^{n_{r}}=: \xi:^{n} .
\end{aligned}
$$

This shows the result.

Corollary 1. If $\xi_{1} \in H_{\infty-}$ and if $\xi_{2} \in H_{\infty-}$

$$
\phi_{\xi_{1}+\xi_{2}}=: \phi_{\xi_{1}} \phi_{\xi_{2}}: \text {. }
$$


Definition 4. Let $\Xi$ belong to $L\left(W_{\infty_{-}}^{r}, W_{\infty_{-}}\right)$. Its Hochschild coboundary $\delta^{r}$ is defined as follows:

$$
\begin{aligned}
\delta^{r} \Xi & \left(\phi^{1}, \ldots, \phi^{r+1}\right)=: \phi^{1} \Xi\left(\phi^{2}, \ldots, \phi^{r}\right) \\
& +\sum_{i=1}^{r}(-1)^{i} \Xi\left(\phi^{1}, \ldots,: \phi^{i} \phi^{i+1}:, \ldots, \phi^{r+1}\right)+(-1)^{r+1}: \Xi\left(\phi^{1}, \ldots, \phi^{r}\right) \phi^{r+1}: .
\end{aligned}
$$

Classically $\delta^{r+1} \delta^{r}=0$.

Definition 5. We say that an element $\Xi$ of $L\left(W_{\infty_{-}}^{r}, W_{\infty_{-}}\right)$is a homogeneous polydifferential operator of order $(l, m)$ if its symbol $\hat{\Xi}\left(\xi^{1}, \ldots, \xi^{r}, \eta\right)$ is equal to

$$
\Psi\left(\xi^{1}, \ldots, \xi^{r}, \eta\right) \exp \left[\sum\left\langle\xi^{i}, \eta\right\rangle\right]
$$

where $\Psi$ is a homogeneous polynomial in the $\xi^{i}$ of degree $m$ and in $\eta$ of degree $l$.

Proposition 1. If $\Xi$ is an $r$-polydifferential operator of degree $(l, m), \delta^{r} \Xi$ is an $(r+1)$-polydifferential operator of degree $(l, m)$.

Proof. Since : $\phi_{\xi^{1}} \phi_{\xi^{2}}:=\phi_{\xi^{1}+\xi^{2}}$, the only problem is to show that

$$
\left(\phi^{1}, \ldots, \phi^{r+1}\right) \rightarrow: \phi^{1} \Xi\left(\phi^{2}, \ldots, \phi^{r+1}\right):
$$

is still a polydifferential operator of degree $(l, m)$.

Let $\eta \in H_{\infty-}$ be such that $\|\eta\|_{0}=1$. Let us compute

$$
\left.\left\langle\Xi\left(\phi_{\xi^{2}}, \ldots, \phi_{\xi^{r+1}}\right), \phi_{\lambda \eta}\right)\right\rangle_{0}=\Psi\left(\xi^{2}, \ldots, \xi^{r+1}, \lambda \eta\right) \exp \left[\lambda \sum_{i=2}^{r+1}\left\langle\xi^{i}, \eta\right\rangle\right] .
$$

If we compute the component of $\Xi\left(\phi_{\xi^{2}}, \ldots, \phi_{\xi^{r+1}}\right)$ along $\frac{\eta^{\otimes n}}{n !}$, it is the element of degree $n$ in the expansion in $\lambda$ of the (3). Since $\Psi$ is homogeneous of degree $l$ in $\eta$, the term of degree $n$ in the expansion in $\lambda$ of (3) is

$$
\frac{\sum_{i=2}^{r+1}\left\langle\xi_{i}, \eta\right\rangle^{n-l}}{n-l !} C_{l}\left(\xi^{2}, \ldots, \xi^{r+1}, \eta\right)
$$

Because the component of $\phi_{\xi^{1}}$ along $\frac{\eta^{\otimes n}}{n !}$ is $\frac{\left\langle\xi^{1}, \eta\right\rangle^{n}}{n !}$, this shows that the component of

$$
: \phi_{\xi^{1}} \Xi\left(\phi_{\xi^{2}}, \ldots, \phi_{\xi^{r+1}}, \lambda \eta\right):
$$

along $\frac{\eta^{\otimes n}}{n !}$ is

$$
C_{l}\left(\xi^{2}, \ldots, \xi^{r+1}, \eta\right) \sum_{n_{1}+n_{2}=n} \frac{\left\langle\sum_{i=2}^{r+1} \xi^{i}, \eta\right\rangle^{n_{1}-l}}{\left(n_{1}-l\right) !} \frac{\left\langle\xi^{1}, \eta\right\rangle^{n_{2}}}{n_{2} !}=C_{l}\left(\xi^{2}, \ldots, \xi^{r+1}, \eta\right) \frac{\left\langle\sum_{i=1}^{r+1} \xi_{i}, \eta\right\rangle^{n-l}}{(n-l) !} .
$$

The result follows directly.

Definition 6. The continuous Hochschild cohomology $H_{\text {cont }}^{r}\left(W_{\infty_{-}}, W_{\infty_{-}}\right)$of the Hida test algebra is the space $\operatorname{Ker} \delta^{r} / \operatorname{Im} \delta^{r-1}$, where the Hochschild coboundary acts on $L\left(W_{\infty-}^{r}, W_{\infty_{-}}\right)$. 
We consider cochains which are finite sums of polydifferential operators of degree $(l, m) \in$ $(\mathbb{N} \times \mathbb{N})$. We call the space of polydifferential operators $L_{\text {dif }}\left(W_{\infty_{-}}^{r}, W_{\infty_{-}}\right)$. By the previous proposition, $\delta^{r}$ applies $L_{\text {dif }}\left(W_{\infty_{-}}^{r}, W_{\infty_{-}}\right)$into $L_{\text {dif }}\left(W_{\infty_{-}}^{r+1}, W_{\infty_{-}}\right)$.

Definition 7. The differential Hochschild cohomology $H_{\text {dif }}^{r}\left(W_{\infty_{-}}, W_{\infty_{-}}\right)$of the Hida test algebra is the space $\operatorname{Ker} \delta^{r} / \operatorname{Im} \delta^{r-1}$ where $\delta^{r}$ acts on $L_{\mathrm{dif}}\left(W_{\infty_{-}}^{r}, W_{\infty_{-}}\right)$.

We get the main theorem of this work:

Theorem 3. The differential Hochschild cohomology groups of the Hida test algebra are equal to the continuous Hochschild cohomology groups of the Hida test algebra.

Proof. This comes from the Fock expansion of the previous part and from the following fact: if $\delta \Xi$ is a polydifferential operator for a continuous cochain $\Xi$, there exists a polydifferential operator $\Xi_{1}$ such that $\delta \Xi=\delta \Xi_{1}$ by Proposition 1 .

\section{Acknowledgements}

Author thank L. Accardi and G. Pinczon for helpful discussions.

\section{References}

[1] Albeverio S., Høegh-Krohn R., Dirichlet forms and diffusion processes on rigged Hilbert spaces, Z. Wahrscheinlichkeitstheorie und Verw. Gebiete 40 (1977), 1-57.

[2] Bayen F., Flato M., Fronsdal C., Lichnerowicz A., Sternheimer D., Deformation theory and quantization. I. Deformations of symplectic structures, Ann. Physics 111 (1978), 61-110.

[3] Bayen F., Flato M., Fronsdal C., Lichnerowicz A., Sternheimer D., Deformation theory and quantization. II. Physical applications, Ann. Physics 111 (1978), 111-151.

[4] Berezansky Y.M., Kondratiev Y.G., Spectral methods in infinite-dimensional analysis, Vols. I, II, Kluwer Academic Publishers, Dordrecht, 1995.

[5] Berezin F.A., The method of second quantization, Academic Press, New York, 1966.

[6] Berezin F.A., Wick and anti-Wick symbols of operators, Mat. Sb. (N.S.) 86(128) (1971), 578-610.

[7] Chung D.M., Ji U.C., Obata N., Higher powers of quantum white noises in terms of integral kernel operators, Infin. Dimens. Anal. Quantum Probab. Relat. Top. 1 (1998), 533-559.

[8] Connes A., Noncommutative differential geometry, Inst. Hautes Études Sci. Publ. Math. (1985), no. 62, $257-360$.

[9] Di Francesco P., Mathieu P., Sénéchal D., Conformal field theory, Springer-Verlag, New York, 1997.

[10] Dito G., Star-product approach to quantum field theory: the free scalar field, Lett. Math. Phys. 20 (1990), $125-134$.

[11] Dito G., Star-products and nonstandard quantization for Klein-Gordon equation, J. Math. Phys. 33 (1992), 791-801.

[12] Dito G., Deformation quantization on a Hilbert space, in Noncommutative Geometry and Physics (Yokohama, 2004), Editors Y. Maeda and et al., World Sci. Publ., Hackensack, NJ, 2005, 139-157, math.QA/0406583.

[13] Dito G., Léandre R., Stochastic Moyal product on the Wiener space, J. Math. Phys. 48 (2007), 023509, 8 pages.

[14] Dütsch M., Fredenhagen K., Perturbative algebraic field theory and deformation quantization, in Mathematical Physics in Mathematics and Physics (Siena, 2000), Fields Inst. Commun., Vol. 30, Amer. Math. Soc., Providence, RI, 2001, 151-160, hep-th/0101079.

[15] Gerstenhaber M., Schack S.D., Algebraic cohomology and deformation theory, in Deformation Theory of Algebras and Structures and Application (Il Ciocco, 1986), NATO Adv. Sci. Inst. Ser. C Math. Phys. Sci., Vol. 247, Editors M. Hazewinkel and M. Gerstenhaber, Kluwer Acad. Publ., Dordrecht, 1988, 11-264.

[16] Glimm J., Jaffe A., Boson quantum fields, in Collected Papers. I, Birkhäuser, Basel, 1985, 125-199. 
[17] Haag R., On quantum field theory, Danske Vid. Selsk. Mat.-Fys. Medd. 29 (1955), no. 12, 37 pages.

[18] Hida T., Analysis of Brownian functionals, Carleton Mathematical Lecture Notes, no. 13, Carleton Univ., Ottawa, Ont., 1975.

[19] Hida T., Kuo H.H., Potthoff J., Streit L., White noise: an infinite-dimensional calculus, Kluwer Academic Publishers, Dordrecht, 1993.

[20] Ji U.C., Obata N., A unified characterization theorem in white noise analysis, Infin. Dimens. Anal. Quantum Probab. Relat. Top. 6 (2003), 167-178.

[21] Ji U.C., Obata N., Quantum white noise calculus, in Non-Commutativity, Infinite-Dimensionality and Probability at the Crossroads, Quantum Probab. White Noise Anal., Vol. 16, Editors N. Obata, T. Matsui and A. Hora, World Sci. Publ., River Edge, NJ, 2002, 143-191.

[22] Léandre R., Wiener analysis and cyclic cohomology, in Stochastic Analysis and mathematical physics (Santiago, 2001), Editors R. Rebolledo and J.C. Zambrini, World Sci. Publ., River Edge, NJ, 2004, 115-127.

[23] Léandre R., Deformation quantization in white noise analysis, in Geometric Aspects of Integrable Systems (Coimbra, 2006), Editor J.P. Françoise et al., SIGMA 3 (2007), 027, 8 pages, math.QA/0702624.

[24] Léandre R., Fedosov quantization in white noise analysis, in NEEDS 2007 (Almetla del Mar, 2007), Editors J. Puig et al., J. Nonlinear Math. Phys., to appear.

[25] Léandre R., Deformation quantization in infinite-dimensional analysis, in Festchrift in Honour of H.V. Weizsaecker (Kaiserslautern, 2007), Editors M. Scheutzow et al., to appear.

[26] Léandre R., Rogers A., Equivariant cohomology, Fock space and loop groups, J. Phys. A: Math. Gen. 39 (2006), 11929-11946.

[27] Maassen H., Quantum Markov processes on Fock space described by integral kernels, in Quantum Probability and Applications, II (Heidelberg, 1984), Lecture Notes in Math., Vol. 1136, Springer, Berlin, 1985, 361-374.

[28] Malliavin P., Stochastic calculus of variations and hypoelliptic operators, in Proceedings of the International Symposium on Stochastic Differential Equations (Res. Inst. Math. Sci., Kyoto Univ., Kyoto, 1976), Editor K. Itô, Wiley, New York - Chichester - Brisbane, 1978, 195-263.

[29] Meyer P.-A., Elements de probabilites quantiques. I-V, in Séminaire de Probabilites, XX, 1984/85, Lecture Notes in Math., Vol. 1204, Editors J. Azéma and M. Yor, Springer, Berlin, 1986, 186-312.

[30] Meyer P.-A., Distributions, noyaux, symboles d'apres Krée, in Seminaire de Probabilites, XXII, Lecture Notes in Math., Vol. 1321, Editors J. Azéma and M. Yor, Springer, Berlin, 1988, 467-476.

[31] Meyer P.-A., Quantum probability for probabilists, Lecture Notes in Mathematics, Vol. 1538, SpringerVerlag, Berlin, 1993.

[32] Nadaud F., On continuous and differential Hochschild cohomology, Lett. Math. Phys. 47 (1999), 85-95.

[33] Nadaud F., Déformations et déformations généralisées, These, Université de Bourgogne, 2000.

[34] Obata N., An analytic characterization of symbols of operators on white noise functionals, J. Math. Soc. Japan 45 (1993), 421-445.

[35] Obata N., White noise analysis and Fock space, Lecture Notes in Mathematics, Vol. 1577, Springer-Verlag, Berlin, 1994.

[36] Pflaum M., On continuous Hochschild homology and cohomology groups, Lect. Math. Phys. 44 (1998), $43-51$.

[37] Pinczon G., On the equivalence between continuous and differential deformation theories, Lett. Math. Phys. 89 (1997), 143-156.

[38] Pinczon G., Ushirobira R., Supertrace and superquadratic Lie structure on the Weyl algebra, and applications to formal inverse Weyl transform, Lett. Math. Phys. 74 (2005), 263-291, math.RT/0507092.

[39] Streit L., An introduction to white noise analysis, in Stochastic Analysis and Applications in Physics (Funchal, 1993), NATO Adv. Sci. Inst. Ser. C Math. Phys. Sci., Vol. 449, Editors A.I. Cardoso et al., Kluwer Acad. Publ., Dordrecht, 1994, 415-439.

[40] Witten E., Noncommutative geometry and string field theory, Nuclear Phys. B 268 (1986), 253-294. 